\title{
Decrescent intensity training concurrently improves maximal anaerobic power, maximal accumulated oxygen deficit, and maximal oxygen uptake
}

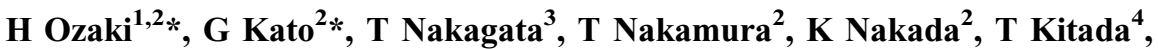 \\ S Katamoto ${ }^{2}$, H Naito ${ }^{2}$ \\ ${ }^{1}$ School of Sport and Health Science, Tokai Gakuen University, Miyoshi, Japan \\ ${ }^{2}$ Graduate School of Health and Sports Science, Juntendo University, Inzai, Japan \\ ${ }^{3}$ Sportology Center, Juntendo University Graduate School of Medicine, Bunkyo-ku, Japan \\ ${ }^{4}$ Institute of Innovation for Future Society, Nagoya University, Nagoya, Japan
}

Received: July 30, 2019

Accepted: October 30, 2019

\begin{abstract}
This study aimed to investigate the effects of a gradually decreasing intensity training from that corresponding to maximal anaerobic power (MAnP) to that of near maximal oxygen uptake $\left(\dot{V}_{2 \max }\right)$ (decrescent intensity training) on MAnP, maximal accumulated oxygen deficit (MAOD), and $\dot{V} \mathrm{O}_{2 \max }$ in untrained young men. Seventeen untrained young men were randomly divided into either a training (TR; $n=9)$ group or a control $(\mathrm{CON} ; n=8)$ group. The TR group performed the decrescent intensity training, whereas the CON group did not perform any exercises. The mean training time per session throughout the training period was $275 \pm 135 \mathrm{~s}$. There was a Group $\times$ Time interaction for both absolute and relative $(p<0.01)$ values of $\dot{V} \mathrm{O}_{2 \max }$, MAOD, and MAnP. The TR group had significantly increased values for all variables after the 8-week training program, and the relative values of all variables were significantly higher in the TR group than in the CON group. Muscle thicknesses in the anterior and posterior aspects of the thigh and maximal isokinetic knee extension and flexion strengths improved only in the TR group $(p<0.05)$. A single-exercise training with gradually decreasing intensity from that corresponding to the MAnP to that of approximately $100 \% \dot{V} \mathrm{O}_{2 \max }$ improves MAnP, MAOD, and $\dot{V} \mathrm{O}_{2 \max }$ concurrently, despite the short training time per session.
\end{abstract}

Keywords: maximal oxygen uptake, anaerobic capacity, anaerobic power, training, adaptation

\section{Introduction}

Adenosine triphosphate (ATP) is resynthesized from anaerobic and aerobic metabolic energy sources during short-duration, high-intensity exercise $(2,9)$. These sources have specific capacity and rate limitations, which may limit performance in many sports $(6,9,13)$. The maximum rate of oxygen consumption (aerobic metabolic system) is conventionally evaluated by maximal oxygen uptake $\left(\dot{V} \mathrm{O}_{2 \max }\right)(15)$; however, it is proposed that the maximal amount of ATP resynthesized via the anaerobic metabolism (maximal anaerobic capacity) is evaluated by maximal accumulated oxygen deficit (MAOD) (12). For example, the energy required during repeated sprint observed in many sports, such as soccer and handball, is supplied from both aerobic and anaerobic metabolic systems, and high $\dot{V} \mathrm{O}_{2 \max }$ and MAOD

\footnotetext{
* These authors equally contributed to this work.

Corresponding author: Hayao Ozaki

School of Sport and Health Science, Tokai Gakuen University

21-233 Nishinohora, Ukigai, Miyoshi, Aichi, Japan

Phone: +81 56136 5555; Fax: +81 56136 6757; E-mail: ozaki.hayao@gmail.com
} 
help maintain sprint performance (6). The maximal anaerobic power (MAnP) assessed by the short all-out sprint is assumed to be the expression of the maximal rate of anaerobic ATP synthesis (3). Almost all of the energy required during the initial several seconds of maximal effort exercise are supplied from anaerobic metabolism system. The rate of energy release is critical to the success of athletic movements, such as sprint, that require the development and short-term maintenance of high power outputs (5). Thus, developing a training method for effectively improving these abilities ( $\dot{V} \mathrm{O}_{2 \max }, \mathrm{MAOD}$, and MAnP) is important for enhancing sport performance. For stimulating aerobic and/or anaerobic metabolism systems, continuous or high-intensity interval training is extensively used $(7,10,20)$, but no previous studies have demonstrated concurrent improvements in all three abilities by a training intervention with only a single exercise (20).

Based on one's specific adaptation to imposed demands, if a single exercise includes intensive stimuli for the rate of oxygen consumption and anaerobic ATP synthesis, as well as anaerobic capacity, it can enhance all three abilities concurrently. Recently, we demonstrated that a single high-load [ $80 \%$ of one repetition maximum $(1 \mathrm{RM})]$ set with additional drop sets descending to a low load (30\% 1RM), that is, the simultaneous execution of both high and low loads, without recovery intervals, can simultaneously increase muscle strength (1RM) and endurance (maximum repetitions at $30 \% 1 \mathrm{RM}$ ) in untrained young men (14). Training with high and low loads produces strong results in the test under high- and low-load conditions, respectively (17): Adaptations in muscle strength and endurance are consistent with the principle of specificity. From a viewpoint of the metabolic demands of training, MAnP required the highest and $\dot{V} \mathrm{O}_{2 \max }$ required the lowest exercise intensity for improvements. Thus, we hypothesized that, based on the method of drop sets training, the decrescent intensity training from an exercise intensity that corresponds to MAnP to that of near $\dot{V} \mathrm{O}_{2 \max }$ improves the rate of oxygen consumption and anaerobic ATP synthesis as well as anaerobic capacity.

The aims of this study were to clarify whether a gradually decreasing exercise intensity training (decrescent intensity training) from that which corresponds to MAnP to that of near $\dot{V} \mathrm{O}_{2 \max }$ concurrently improves MAnP, MAOD, and $\dot{V} \mathrm{O}_{2 \max }$ in untrained young men. Because it is difficult to differentiate the effect of this training program from those of other exercise trainings in studies employing athletes, we enrolled untrained subjects.

\section{Methods}

\section{Subjects}

Seventeen untrained young men volunteered to participate in the study. Subjects were recruited through printed advertisements and by word-of-mouth. No subjects had participated in any regular endurance and resistance training for 1 year prior to enrollment in the study. Past or present smokers and anyone taking any medications and/or supplements were excluded. All subjects were informed of the methods, procedures, and risks, and they signed an informed consent document before participating in the study. This study was conducted according to the principles of the Declaration of Helsinki and was approved by the Ethics Committee for Human Experiments of Juntendo University, Japan. 


\section{Study design}

Each subject visited the laboratory five separate times for measurements before the start of the training period (PRE). On the first day, the muscle thickness (MT) of their thighs was measured, and they underwent maximal exercise testing on an electromagnetically braked cycle ergometer (Powermax V2, Combi, Tokyo, Japan) to determine $\dot{V} \mathrm{O}_{2 \max }$. On the second and third days, they participated in submaximal exercise tests to determine a linear relationship between the submaximal intensity of cycling (watts) and the steady-state oxygen uptake $(\mathrm{L} / \mathrm{min})$. On the fourth day, the subjects were familiarized with the maximal isometric and isokinetic strength tests using a System 3 dynamometer (Biodex, Shirley, NY, USA), which were taken on the fifth day, and they participated in a supramaximal exercise test on a cycle ergometer that exhausted subjects within 2-3 min to calculate MAOD. $\dot{V} \mathrm{O}_{2}$ was measured using an automated metabolic cart (Aeromonitor AE-300S, Minato Medical Science, Tokyo, Japan) during the maximal and submaximal exercise tests. In the supramaximal exercise test, the Douglas bag method was used for the accurate calculation of MAOD. Gas volume was measured by a gasometer (Shinagawa Seisakusho, Tokyo, Japan), and fractions of oxygen and carbon dioxide in the expired air were measured by the metabolic cart. On the fifth day, they underwent maximal strength test and then maximal effort sprint tests at three different loads to determine MAnP (11). The familiarization session of the training protocol was performed 2-3 days before the training period. After 4 weeks of training, MAnP and $\dot{V} \mathrm{O}_{2 \max }$ were evaluated in a single day (MID). The $\dot{V} \mathrm{O}_{2 \max }$ test was performed more than 30 min after the end of the MAnP test. After the training periods, the subjects completed all measurements within 12 days (POST).

\section{Training program}

Subjects were randomly divided into either a training (TR; $n=9)$ group or a control $(\mathrm{CON}$; $n=8$ ) group. The TR group performed the decrescent intensity training on an ergometer, whereas the CON group did not perform any exercises. Subjects were instructed to avoid other physical activities and not change their dietary patterns throughout the course of the study. Before the start of each training session, subjects performed $10 \mathrm{~min}$ of continuous cycling at an intensity of $50 \% \dot{V} \mathrm{O}_{2 \max }$ including three bouts of $5 \mathrm{~s}$ near maximal pedaling in the latter half as a warm-up.

The decrescent intensity training was composed of five consecutive parts without rest intervals between parts, and the exercise intensity gradually decreased by modulating the pedaling rate and load (Fig. 1). Subjects first pedaled with maximal effort for $5 \mathrm{~s}$ at a pedaling load, which corresponds to MAnP (Part I) and then underwent five bouts of $5 \mathrm{~s}$ pedaling (Part II). The pedaling rate decreased by $5 \mathrm{rpm}(85,80,75,70$, and $65 \mathrm{rpm})$ using the load in the supramaximal exercise (MAOD) test in Part II. Immediately thereafter, subjects performed pedaling at $60 \mathrm{rpm}$ at an exercise intensity used in the supramaximal exercise test for 30-60 s (Part III) and then at $105 \% \dot{V} \mathrm{O}_{2 \max }$ until the pedaling rate was under $57 \mathrm{rpm}$ over $3 \mathrm{~s}$ (Part IV). Finally, subjects pedaled at $55 \mathrm{rpm}$ at $90 \% \dot{V} \mathrm{O}_{2 \max }$ until the pedaling rate was under 52 rpm over $3 \mathrm{~s}$ (Part V). The exercise duration in Part III was initially set at $30 \mathrm{~s}$. If subjects maintained the pedaling rate $(60 \mathrm{rpm})$ during the prescribed duration, the exercise duration was extended by $10 \mathrm{~s}$ in the next session. Furthermore, when the pedaling rate was maintained for $60 \mathrm{~s}$ in Part III, in the next session, the exercise intensity increased by $5 \% \dot{V} \mathrm{O}_{2 \max }$ in Parts III-V, and the exercise duration was set at $30 \mathrm{~s}$ in Part III again. After the MID test, the pedaling load in Parts I and II, the relative exercise intensity in Parts III-V was set again based on the results of the MID test, the exercise duration in Part III was reset to 


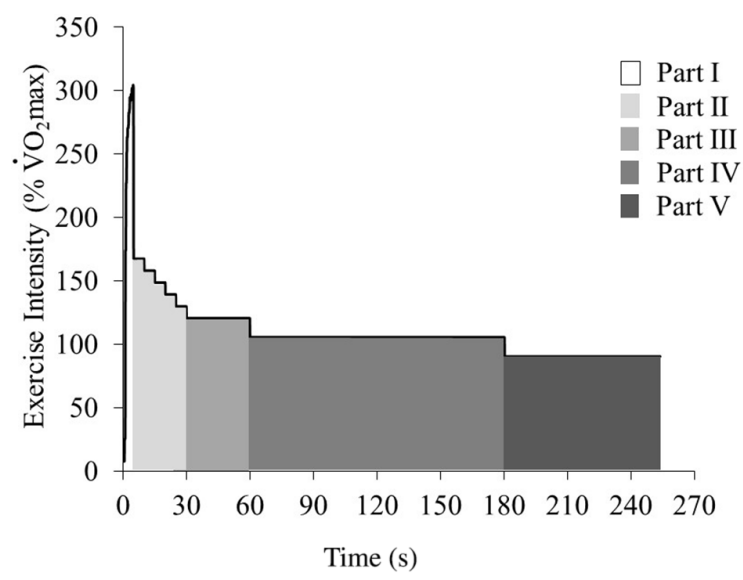

Fig. 1. A typical example of decrescent intensity training. The decrescent intensity training was composed of five consecutive parts without rest intervals between parts, and the exercise intensity gradually decreased by modulating the pedaling rate and load. Subjects first pedaled with maximal effort for $5 \mathrm{~s}$ at a pedaling load, which corresponds to maximal anaerobic power (Part I) and then underwent five bouts of $5 \mathrm{~s}$ pedaling (Part II). The pedaling rate decreased by $5 \mathrm{rpm}(85,80,75,70$, and $65 \mathrm{rpm})$ using the load in the supramaximal exercise test in Part II. Immediately thereafter, subjects performed pedaling at $60 \mathrm{rpm}$ at an exercise intensity used in the supramaximal exercise test for $30-60 \mathrm{~s}$ (Part III) and then at $105 \% \dot{\mathrm{V}} \mathrm{O}_{2 \max }$ until the pedaling rate was under $57 \mathrm{rpm}$ over $3 \mathrm{~s}$ (Part IV). Finally, subjects pedaled at $55 \mathrm{rpm}$ at $90 \% \dot{V} \mathrm{O}_{2 \max }$ until the pedaling rate was under $52 \mathrm{rpm}$ over $3 \mathrm{~s}(\operatorname{Part} \mathrm{V}) . \dot{V} \mathrm{O}_{2 \max }$, maximal oxygen uptake

$30 \mathrm{~s}$, and these variables were then modulated in a similar manner throughout the latter half of the training period. The TR group trained 2 days/week for the first 2 weeks and 3 days/week in the final 6 weeks except on the fifth and eighth weeks. The number of training sessions per week was decreased, so that the subjects can perform the MID tests on the first session of the fifth week and can minimize the effect of fatigue on the POST test.

\section{Maximal exercise test}

The subjects performed a graded maximal exercise test on a cycle ergometer to determine $\dot{V} \mathrm{O}_{2 \text { max }}$. The pedaling rate was maintained constant at $60 \mathrm{rpm}$, and the load was initially set at $1.0 \mathrm{kp}$ preceded by rest in a seated position for $2 \mathrm{~min}$ and increased by $0.3 \mathrm{kp}$ every minute until exhaustion. Each subject's electrocardiograph was monitored throughout and used to measure heart rate (HR) at 1-min intervals. The criteria for $\dot{V} \mathrm{O}_{2 \max }$ used in this study were as follows: (1) no increase in $\dot{V} \mathrm{O}_{2}$ despite increased pedaling load, (2) $\mathrm{HR}>90 \%$ of agepredicted maximum HR (220-age), (3) respiratory exchange ratio greater than 1.10, (4) blood lactate greater than $10 \mathrm{mmol} / \mathrm{L}$, and (5) ratings of perceived exertion greater than 18 . Of these criteria, at least three had to be met to be considered a valid $\dot{V} \mathrm{O}_{2 \max }$, and all subjects met this requirement.

\section{Submaximal exercise test}

The subjects performed submaximal exercise tests on a cycle ergometer to determine a linear relationship between the submaximal intensity of cycling (watts) and the steady-state oxygen uptake $(\mathrm{L} / \mathrm{min})$. Ten different 4 -min bouts were performed at a constant power $(60 \mathrm{rpm})$ between $30 \%$ and $90 \% \dot{V} \mathrm{O}_{2 \max }$ based on a previous research (12). Subjects performed five bouts per day for 2 days. 
Supramaximal exercise test

Subjects participated in a supramaximal exercise test that exhausted them within 2-3 min, by which MAOD can be calculated. The pedaling rate was maintained constant at $60 \mathrm{rpm}$. The oxygen demands during this test were estimated by extrapolating the established relationship between power and steady-state oxygen uptake described above. The accumulated oxygen demand was taken as the product of the estimated oxygen demand and the exercise duration, whereas the accumulated oxygen was taken as the measured oxygen uptake integrated over the exercise duration. The accumulated oxygen deficit (AOD) was calculated as the difference between these two entities, which was reported as MAOD.

\section{Maximal effort sprint tests}

Subjects were instructed to pedal as rapidly as possible in a seated position for $8 \mathrm{~s}$ at three different loads to determine MAnP. The loads (kp) corresponded to 5\%, 10\%, and $15 \%$ of their body mass $(\mathrm{kg})$. Tests were performed in a load-increasing order with rest intervals of more than $5 \mathrm{~min}$. From three different loads and cadences in each subject, the relationship between load and cadence was represented by linear regression equation for each subject $(Y=-a X+b, a>0, b>0)$. Power output in each load was calculated using the following formula:

$$
\text { Power output }(\mathrm{W})=\operatorname{Load}(\mathrm{kp}) \times \text { Cadence }(\mathrm{rpm}) \times 0.98 \text {. }
$$

After calculating the power output in each load, MAnP was determined for each subject based on the linear regression equation for three pairs of loads and cadences described by Nakamura et al. (11).

\section{Muscle thickness (MT)}

MT was measured using a B-mode ultrasound using a 5- to $18-\mathrm{MHz}$ scanning head (Noblus; Aloka, Tokyo, Japan) in six locations: anterior and posterior aspects of the right thigh (AT and PT, respectively) at $40 \%, 50 \%$, and $60 \%$ of the thigh's length between the lateral condyle of the femur and the greater trochanter. Before all scans, the subjects rested in the supine position for at least $30 \mathrm{~min}$. To avoid the influence of fluid shifts within the muscle, the measurements were performed at about the same time. All measurements were performed by the same operator, with participants in the supine/prone position. The subcutaneous adipose tissue-muscle interface and muscle-bone interface were identified on ultrasound images, and the distance between the two interfaces was recorded as the MT. The mean of the MT of the three anterior and posterior sites was used in the data analysis.

\section{Maximum isometric and isokinetic strengths}

Maximum voluntary isometric and isokinetic contractions of the knee extensors (KE) and flexors (KF) were determined using a System 3 dynamometer following the manufacturer's recommended guidelines for positioning. During the test, the subject was seated on a chair with the hip joint angle positioned at $85^{\circ}$ of flexion (full hip extension: $0^{\circ}$ ). Subjects were secured by straps to minimize extraneous and compensatory movements. The knee joint center of rotation was visually aligned with the axis of the dynamometer's lever arm. A knee joint angle of $0^{\circ}$ corresponded to full knee extension. Several warm-up contractions (4-5 submaximal contractions and 2-3 near-maximal contractions at $270 \%$ s) were performed 
before testing. Subjects were then instructed to perform maximal isokinetic knee extension and flexion from $10^{\circ}$ to $100^{\circ}$ at $90 \% \mathrm{~s}, 180^{\circ} / \mathrm{s}$, and $270^{\circ} / \mathrm{s}$. Then, they performed maximal isometric knee extension and flexion $(0 \% \mathrm{~s})$ for about $5 \mathrm{~s}$ at a fixed knee joint angle of $60^{\circ}$ and $15^{\circ}$, respectively. Two maximal efforts were performed. The highest value was used for data analysis.

\section{Statistical analyses}

Statistical tests were performed using SPSS version 23.0 software (SPSS Inc., Chicago, IL, USA). The results are expressed as means and standard deviations. Changes in scores are represented by means and $95 \%$ confidence intervals. For $\dot{V} \mathrm{O}_{2 \max }$ and MAnP, we used repeated measures analysis of variance (ANOVA) on time with a between-subject factor of group. If there was a significant interaction, Bonferroni adjusted post-hoc tests were used across time within each group and a $t$-test was used across groups within each time point. For MAOD, repeated measures ANOVA on time with a between-subject factor of group was used. If there was a significant interaction, post-hoc tests were performed by a $t$-test. Statistical significance was set at $p<0.05$.

\section{Results}

All subjects completed the study, and the overall adherence was good, with a participation rate of $100 \%$ in the TR group. No significant differences between conditions were evident in any baseline values (Table I). The mean training time per session throughout the training period was $275 \pm 135 \mathrm{~s}$. Those from 1st to 10th sessions and from 11th to 20th sessions were $375 \pm 264 \mathrm{~s}$ and $175 \pm 53 \mathrm{~s}$, respectively.

\section{$\dot{V} \mathrm{O}_{2 \max }$}

There was a Group $\times$ Time interaction for both absolute $(p<0.001)$ and relative $(p=0.001)$ values of $\dot{V} \mathrm{O}_{2 \max }$ (Fig. 2A, B). For the absolute value, there were no significant differences between groups at PRE $(p=0.986)$ and MID $(p=0.235)$, but the value in the TR group was significantly higher at POST $(p=0.042)$ than that in CON. Across time, the TR condition significantly increased from PRE to MID [256.9 $(81.4,432.4) \mathrm{ml} / \mathrm{min}, p=0.004]$ and from MID to POST $[214.8(48.5,381.0) \mathrm{ml} / \mathrm{min}, p=0.010]$. For the relative value, there were no significant differences between the groups at PRE $(p=0.331)$, but the value in the TR group was significantly higher at MID $(p=0.048)$ and POST $(p=0.005)$ than that in the CON group. Across time, the TR condition significantly increased from PRE to MID [3.5 $(0.9,6.1)$ $\left.\mathrm{ml} \cdot \mathrm{min}^{-1} \cdot \mathrm{kg}^{-1}, p=0.008\right]$ and from MID to POST $\left[3.1(0.6,5.6) \mathrm{ml} \cdot \mathrm{min}^{-1} \cdot \mathrm{kg}^{-1}\right.$, $p=0.012]$.

There was a Group $\times$ Time interaction for the exercise time in the maximal exercise test $(p=0.001)$. There were no significant differences between groups at PRE $(p=0.537)$, MID $(p=0.062)$, and POST $(p=0.060)$. Across time, the TR condition significantly increased from PRE to POST [99.1 $(53.7,144.5) \mathrm{s}, p<0.001]$ and PRE to MID $[75.0(37.4,112.6) \mathrm{s}$, $p<0.001]$ but not MID to POST [24.1 $(-23.6,71.8) \mathrm{s}, p=0.580]$.

\section{$M A O D$}

There was a Group $\times$ Time interaction for both absolute $(p=0.024)$ and relative $(p=0.044)$ values of MAOD (Fig. 3A, B). For both values, there were no significant differences between 


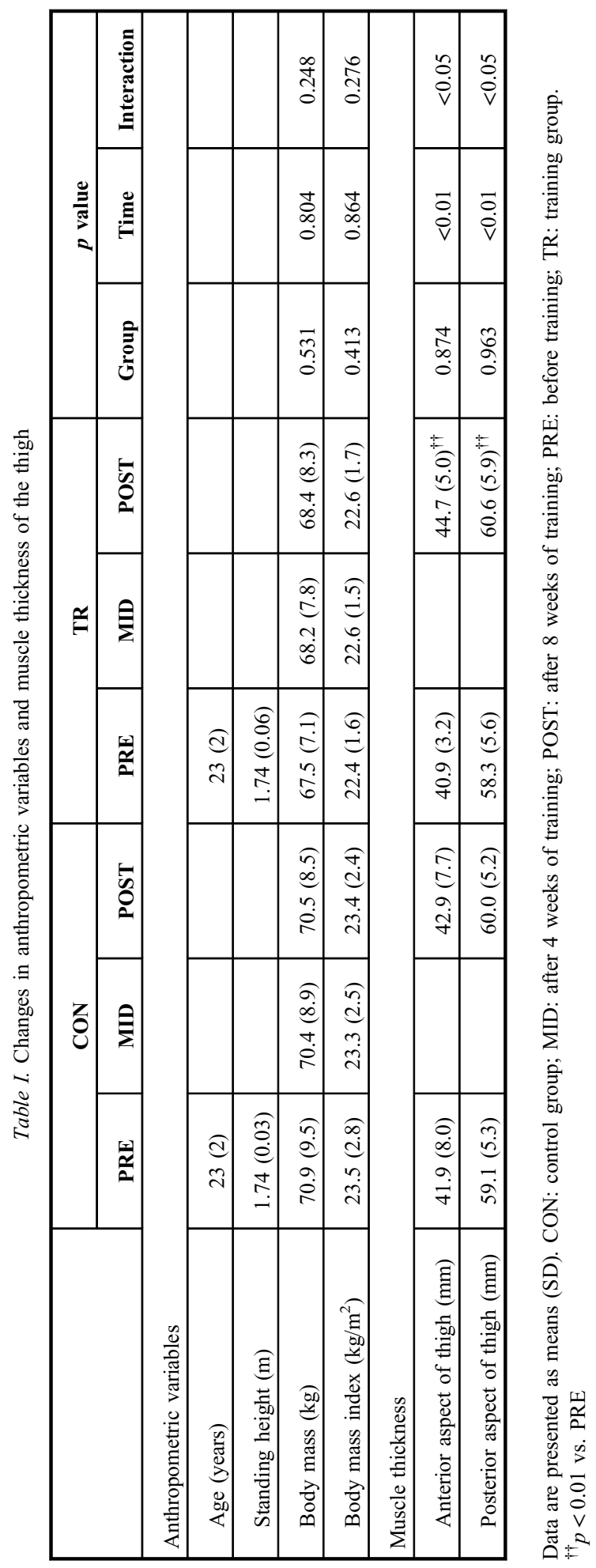



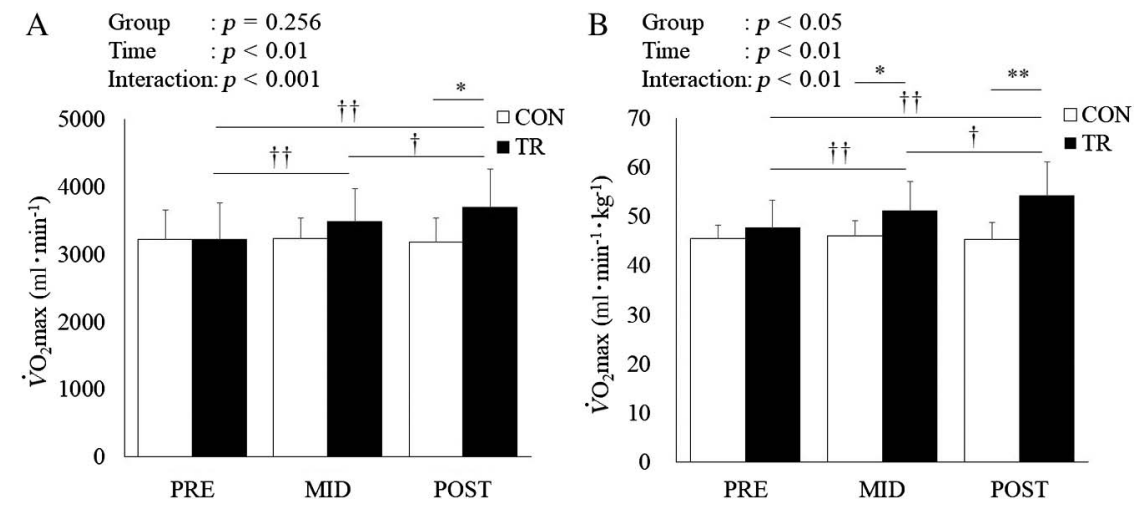

Fig. 2. Changes in $\dot{V} \mathrm{O}_{2 \max }$. Data are presented as means $\pm \mathrm{SD}$. (A) Absolute value. There were no significant differences between groups at PRE and MID, but the value in the TR group was significantly higher at POST than that in CON. Across time, the TR condition significantly increased from PRE to MID and from MID to POST. (B) Relative value. There were no significant differences between the groups at PRE, but the value in the TR group was significantly higher at MID and POST than that in the CON group. Across time, the TR condition significantly increased from PRE to MID and from MID to POST. CON: control group; MID: after 4 weeks of training; POST: after 8 weeks of training; PRE: before training; TR: training group; $\dot{V} \mathrm{O}_{2 \max }$ : maximal oxygen uptake. ${ }^{\dagger \dagger} p<0.01 \mathrm{vs}$.

PRE or MID. ${ }^{\dagger} p<0.05$ vs. MID. ${ }^{*} p<0.05$ vs. CON. $* * p<0.01$ vs. CON
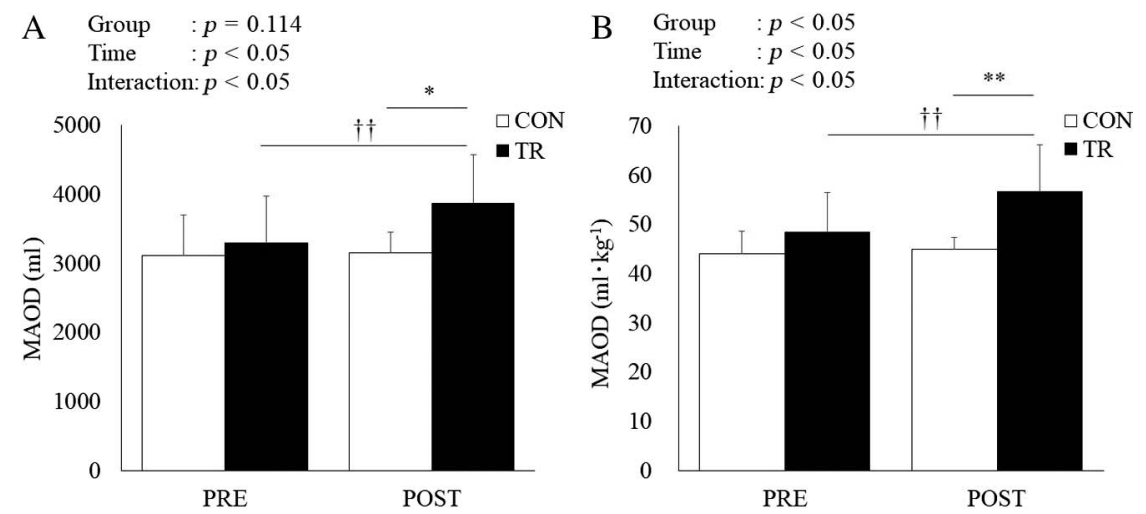

Fig. 3. Changes in MAOD. Data are presented as means \pm SD. (A) Absolute value. (B) Relative value. For both values, there were no significant differences between groups at PRE, but the value in the TR group was significantly higher at POST than that in the CON group. Across time, the TR condition significantly increased from PRE to POST. CON: control group; MAOD: maximal accumulated oxygen deficit; POST: after 8 weeks of training; PRE: before training; TR: training group. ${ }^{\dagger \dagger} p<0.01$ vs. PRE. ${ }^{*} p<0.05$ vs. CON. ${ }^{*} * p<0.01$ vs. CON

groups at PRE (absolute value: $p=0.573$, relative value: $p=0.192$ ), but the value in the TR group was significantly higher at POST (absolute value: $p=0.017$, relative value: $p=0.004$ ) than that in the CON group. Across time, the TR condition significantly increased from PRE to POST [absolute value: $578.3(216.6,895.1) \mathrm{ml}, p=0.001$; relative value: $8.2(3.4,13.0)$ $\mathrm{ml} / \mathrm{kg}, p=0.003]$.

There was a Group $\times$ Time interaction for the exercise load in the supramaximal exercise test $(p<0.001)$. There were no significant differences between groups at PRE $(p=0.928)$ and POST $(p=0.140)$. Across time, the TR condition significantly increased from PRE to POST 
[0.54 $(0.40,0.70) \mathrm{kp}, p<0.001]$. The main effects of group $(p=0.251)$ and time $(p=0.755)$ and Group $\times$ Time interaction $(p=0.520)$ were not significant for the exercise time in the supramaximal exercise test.

\section{$M A n P$}

There was a Group $\times$ Time interaction for both absolute $(p=0.003)$ and relative $(p=0.006)$ values of MAnP (Fig. 4A, B). For the absolute value, there were no significant differences between groups at PRE $(p=0.820), \operatorname{MID}(p=0.804)$, and POST $(p=0.439)$. For the relative value, there were no significant differences between groups at PRE $(p=0.753)$ and MID $(p=0.060)$, but the value in the TR group was significantly higher at POST $(p=0.014)$ than that in the CON group. Across time, the TR condition significantly increased from PRE to POST [absolute value: $64.1(34.2,94.0) \mathrm{W}, p<0.001$, relative value: $0.9(0.4,1.3) \mathrm{W} / \mathrm{kg}$, $p<0.001]$.

There was a Group $\times$ Time interaction for the peak cadence in both $5 \%(p<0.001)$ and $10 \%(p=0.030)$ loads. For the 5\% load, there were no significant differences between groups at PRE $(p=0.581)$, MID $(p=0.966)$, and POST $(p=0.125)$. Across time, the TR condition significantly increased from PRE to POST $[11.8(8.1,15.5) \mathrm{rpm}, p<0.001]$ and PRE to MID $[8.6(5.4,11.9) \mathrm{rpm}, p<0.001]$ but not MID to POST [3.1 $(-1.1,7.3) \mathrm{rpm}, p=0.195]$. For the $10 \%$ load, there were no significant differences between groups at PRE $(p=0.888)$ and $\operatorname{MID}(p=0.243)$, but the value in the TR group was significantly higher at POST $(p=0.044)$ than that in the CON group. Across time, the TR condition significantly increased from PRE to POST $[6.9(1.1,12.7) \mathrm{rpm}, p=0.018]$ but not PRE to MID $[5.6(-0.8,11.9) \mathrm{rpm}$, $p=0.098]$ and MID to POST $[1.3(-2.4,5.1) \mathrm{rpm}, p=1.000]$. The main effects of group $(p=0.103)$ and time $(p=0.165)$ and Group $\times$ Time interaction $(p=0.089)$ were not significant for the peak cadence in $15 \%$ load.
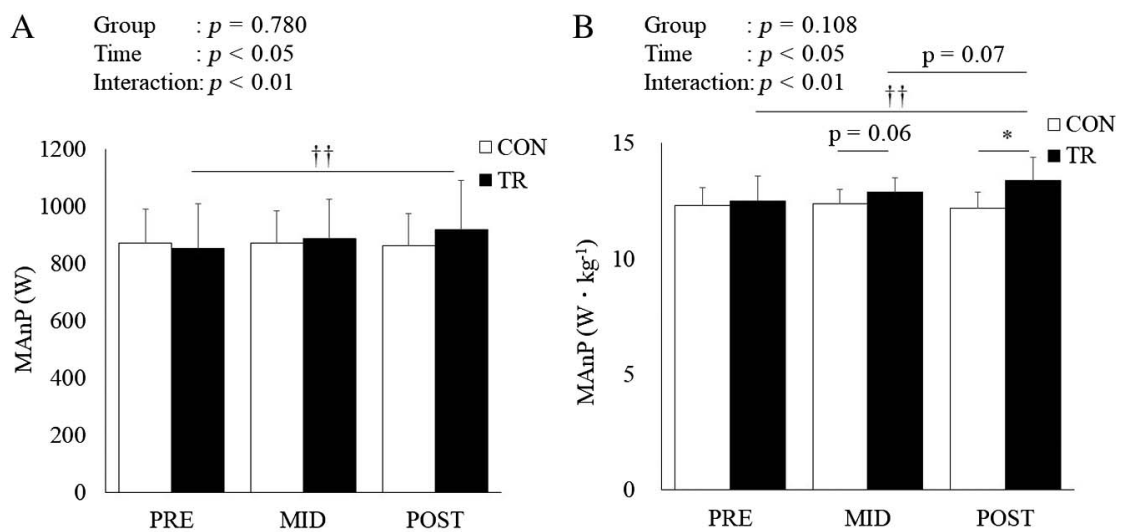

Fig. 4. Changes in MAnP. Data are presented as means \pm SD. (A) Absolute value. There were no significant differences between groups at PRE, MID, and POST. Across time, the TR condition significantly increased from PRE to POST. (B) Relative value. There were no significant differences between groups at PRE and MID, but the value in the TR group was significantly higher at POST than that in the CON group. Across time, the TR condition significantly increased from PRE to POST. CON: control group; MAnP: maximal anaerobic power; MID: after 4 weeks of training; POST: after 8 weeks of training; PRE: before training; TR: training group. ${ }^{\dagger \dagger} p<0.01 \mathrm{vs.}$ PRE. ${ }^{*} p<0.05$ vs. CON 
Table II. Changes in knee extension and flexion strength

\begin{tabular}{|c|c|c|c|c|c|c|c|}
\hline & \multicolumn{2}{|c|}{ CON } & \multicolumn{2}{|c|}{ TR } & \multicolumn{3}{|c|}{$p$ value } \\
\hline & PRE & POST & PRE & POST & Group & Time & Interaction \\
\hline \multicolumn{8}{|c|}{ Knee extension $(\mathrm{Nm})$} \\
\hline $0 \% \mathrm{~s}$ & $245.5(45.1)$ & $239.6(45.2)$ & $268.6(53.5)$ & $266.0(57.5)$ & 0.329 & 0.199 & 0.612 \\
\hline $90 \% / \mathrm{s}$ & $194.5(44.5)$ & $185.2(38.5)$ & $201.4(38.3)$ & $198.5(39.6)$ & 0.607 & 0.119 & 0.397 \\
\hline $180^{\circ} / \mathrm{s}$ & $156.6(32.6)$ & $155.3(32.7)$ & $157.2(29.8)$ & $163.0(28.4)$ & 0.781 & 0.208 & 0.052 \\
\hline $270^{\circ} / \mathrm{s}$ & $130.5(25.0)$ & $128.5(23.4)$ & $129.4(24.6)$ & $136.0(23.2)^{\dagger \dagger}$ & 0.786 & 0.132 & $<0.01$ \\
\hline \multicolumn{8}{|c|}{ Knee flexion $(\mathrm{Nm})$} \\
\hline $0 \% \mathrm{~s}$ & $133.7(33.5)$ & $135.4(33.7)$ & $129.4(25.2)$ & $128.8(34.3)$ & 0.724 & 0.884 & 0.728 \\
\hline $90 \% \mathrm{~s}$ & $95.7(23.1)$ & $97.0(19.8)$ & $89.7(24.4)$ & $95.3(25.5)$ & 0.737 & 0.204 & 0.424 \\
\hline $180^{\circ} / \mathrm{s}$ & $84.5(18.8)$ & $84.5(20.4)$ & $78.2(19.0)$ & $81.8(20.4)$ & 0.640 & 0.232 & 0.223 \\
\hline $270 \% \mathrm{~s}$ & $73.3(16.7)$ & $72.3(14.9)$ & $65.9(17.4)$ & $71.4(18.8)^{\dagger}$ & 0.618 & 0.151 & $<0.05$ \\
\hline
\end{tabular}

Data are presented as means (SD). CON: control group; PRE: before training; POST: after 8 weeks of training; TR: training group.

${ }^{\dagger \dagger} p<0.01$ vs. PRE, ${ }^{\dagger} p<0.05$ vs. PRE

\section{Muscle thickness (MT)}

There was a Group $\times$ Time interaction for MT in AT $(p=0.048)$ and PT $(p=0.047)$ as shown in Table I. For both sites, there were no significant differences between groups at PRE (AT: $p=0.752$; PT: $p=0.765$ ) and POST (AT: $p=0.556$; PT: $p=0.842$ ). Across time, the TR condition significantly increased from PRE to POST [AT: $3.8(1.9,5.7) \mathrm{mm}, p=0.001$, PT: $2.3(1.4,3.2) \mathrm{mm}, p<0.001]$.

\section{Muscle strength}

There was a Group $\times$ Time interaction for maximal isokinetic strengths of knee extension $(p=0.009)$ and flexion $(p=0.042)$ at $270 \%$ s (Table II). For both sites, there were no significant differences between groups at PRE (KE: $p=0.930$; KF: $p=0.385$ ) and POST (KE: $p=0.518$; KF: $p=0.917)$. Across time, the TR condition significantly increased from PRE to POST [KE: $6.6(2.4,10.8) \mathrm{Nm}, p=0.004$; KF: $5.5(1.2,9.8) \mathrm{Nm}, p=0.016]$. The main effects of group $(p=0.329-0.884)$ and time $(p=0.119-0.737)$ as well as Group $\times$ Time interaction $(p=0.052-$ 0.728 ) were not significant for the isokinetic and isometric strength of $\mathrm{KE}$ and KF.

\section{Discussion}

The major finding of this study was that MAnP, MAOD, and $\dot{V} \mathrm{O}_{2 \max }$ were improved concurrently by 8 weeks of a single-exercise training using the decrescent intensity training method from that which corresponds to MAnP to that of near $100 \% \dot{V} \mathrm{O}_{2 \text { max }}$ in untrained young men. Furthermore, these training effects were achieved with short training time per session: the mean value was about $4.5 \mathrm{~min}$. The mean training time in the latter half of the 
training period (from 11 th to 20 th sessions) was about $3 \mathrm{~min}$, because the training intensity increased gradually throughout the training period.

Given the specific adaptation to imposed demands, the intensive stimuli imposed on aerobic and anaerobic energy supplying systems appear important for the improvements in $\dot{V} \mathrm{O}_{2 \max }$ and MAOD. Wenger and Bell (21) demonstrated the greatest improvements in $\dot{V} \mathrm{O}_{2 \max }$ when intensity is from $90 \%$ to $100 \% \dot{V} \mathrm{O}_{2 \max }$. Although the information on the training method for improving MAOD is limited, Tabata et al. (20) demonstrated that the exhaustive intermittent training that consisted of 7-8 sets of 20-s exercise at an intensity of about $170 \% \dot{V} \mathrm{O}_{2 \max }$ with a 10 -s rest between each bout increased $\dot{V} \mathrm{O}_{2 \max }$ and MAOD after the 6-week training period. Furthermore, Kouzaki and Tabata (8) showed that intermittent training that consisted of 6-7 sets of 20 -s exercise at $160 \%-200 \% \dot{V} \mathrm{O}_{2 \max }$ with a 10 -s rest between each bout increased them after the 8-week training period. These studies also showed that the AOD during exercise was not significantly different from MAOD, and the peak $\dot{V} \mathrm{O}_{2}$ during exercise was not significantly less than the $\dot{V} \mathrm{O}_{2 \max }(8,19)$. In the last session of this study, $\dot{V} \mathrm{O}_{2}$ was above $90 \% \dot{V} \mathrm{O}_{2 \max }$ at about $1 \mathrm{~min}$ from the start of the training and then kept at the level of near $\dot{V} \mathrm{O}_{2 \max }$ during the rest of the training duration (data not shown). In addition, because subjects pedaled at an exercise intensity slightly above $\dot{V} \mathrm{O}_{2 \max }$ until exhaustion in Part IV, the AOD from Parts I to IV was roughly comparable to the MAOD. In fact, the mean AOD was about $90 \%$ of MAOD (data not shown), although the variation of energy demand by acceleration and deceleration of pedaling was not made considering its calculation. Thus, imposing intensive stimuli on two energy supplying systems would result in improvements in $\dot{V} \mathrm{O}_{2 \max }$ and MAOD in this study. Meanwhile, with regard to training frequency, Fox et al. (4) demonstrated no difference in the magnitude of the improvement in $\dot{V} \mathrm{O}_{2 \max }$ after high-intensity interval training between 2 and 4 days per week. Thus, the training frequency (2-3 days per week) in this study was sufficient for at least the improvement in $\dot{V} \mathrm{O}_{2 \max }$. Furthermore, given that the aerobic and anaerobic energy production during intense exercise is related to the muscle mass involved $(1,16)$, muscle hypertrophy observed in the TR group would contribute at least partly to the improvements in $\dot{V} \mathrm{O}_{2 \max }$ and MAOD.

MAnP also increased in addition to $\dot{V} \mathrm{O}_{2 \max }$ and MAOD by the decrescent intensity training in this study. To the best of our knowledge, no study has investigated the effect of a cycling training with a cycle ergometer on MAnP, because the Wingate anaerobic test, in which its load is not necessarily equal to that corresponding to MAnP, has been popularized for the evaluation of the anaerobic-type power output $(3,11)$. However, based on the specific adaptation to imposed demands, it is plausible to assume that even only a 5-s maximal effort of pedaling at the load corresponding to MAnP (Part I) contributes to improvement in MAnP in untrained subjects. In addition, the improvement in MAnP resulted from improving the peak cadence in the loads of $5 \%$ and $10 \%$ of the body weight in this study. The mean pedaling loads of Part I in the TR group were 7-8 kp throughout the training period, which corresponded to $10 \%-11 \%$ of their body weight. This means that the peak cadence improved in the load conditions, which corresponded to and under the mean pedaling loads in the training sessions. Furthermore, maximal knee extension and flexion strengths significantly improved only at the fastest condition $\left(270^{\circ} / \mathrm{s}\right)$. This may be because this study used the higher intensity (speed) training, given that knee extension strengths at the lower test speeds $\left(30^{\circ}, 60^{\circ}\right.$, and $\left.120^{\circ} / \mathrm{s}\right)$ increased significantly in the previous study using the lower intensity (speed) training (18). These improvements would contribute at least partly to the increments for the peak cadence in this study. 
In conclusion, this is the first study to show that 8 weeks of a single-exercise training using the decrescent intensity training method from that which corresponds to MAnP to that of near $100 \% \dot{V} \mathrm{O}_{2 \max }$ improves concurrently MAnP, MAOD, and $\dot{V} \mathrm{O}_{2 \max }$, despite the short training time per session. Our training protocol may also afford potential adaptation benefits for athletes and/or enthusiasts with limited exercise time, although this warrants further investigation. Further work is required to define the training protocols necessary to maximize these training effects.

\section{Acknowledgements}

This work was supported by Mizuno Sports Foundation. The authors would like to thank the Juntendo University Institute of Health and Sports Science \& Medicine for supporting this research.

\section{Conflict of interest}

The authors report no conflict of interest.

\section{REFERENCES}

1. Bangsbo J, Michalsik L, Petersen A: Accumulated $\mathrm{O}_{2}$ deficit during intense exercise and muscle characteristics of elite athletes. Int. J. Sports. Med. 14, 207-213 (1993)

2. Beneke R, Boning D: The limits of human performance. Essays Biochem. 44, 11-25 (2008)

3. Driss T, Vandewalle H: The measurement of maximal (anaerobic) power output on a cycle ergometer: a critical review. Biomed. Res. Int. 2013, 589361 (2013)

4. Fox EL, Bartels RL, Billings CE, O’Brien R, Bason R, Mathews DK: Frequency and duration of interval training programs and changes in aerobic power. J. Appl. Physiol. 38, 481-484 (1975)

5. Gastin PB: Energy system interaction and relative contribution during maximal exercise. Sports Med. 31, 725-741 (2001)

6. Girard O, Mendez-Villanueva A, Bishop D: Repeated-sprint ability - Part I: factors contributing to fatigue. Sports Med. 41, 673-694 (2011)

7. Gist NH, Fedewa MV, Dishman RK, Cureton KJ: Sprint interval training effects on aerobic capacity: a systematic review and meta-analysis. Sports Med. 44, 269-279 (2014)

8. Kouzaki M, Tabata I: Effects of high intensity intermittent training on maximal oxygen deficit and maxima oxygen uptake. J. Training Sci. 9, 83-94 (1998) (in Japanese)

9. McMahon S, Jenkins D: Factors affecting the rate of phosphocreatine resynthesis following intense exercise. Sports Med. 32, 761-784 (2002)

10. Milanovic Z, Sporis G, Weston M: Effectiveness of high-intensity interval training (HIT) and continuous endurance training for $\dot{V} \mathrm{O}_{2 \max }$ improvements: a systematic review and meta-analysis of controlled trials. Sports Med. 45, 1469-1481 (2015)

11. Nakamura Y, Mutoh Y, Miyashita M: Determination of the peak power output during maximal brief pedalling bouts. J. Sports Sci. 3, 181-187 (1985)

12. Noordhof DA, de Koning JJ, Foster C: The maximal accumulated oxygen deficit method: a valid and reliable measure of anaerobic capacity? Sports Med. 40, 285-302 (2010)

13. Noordhof DA, Skiba PF, de Koning JJ: Determining anaerobic capacity in sporting activities. Int. J. Sports Physiol. Perform. 8, 475-482 (2013)

14. Ozaki H, Kubota A, Natsume T, Loenneke JP, Abe T, Machida S, Naito H: Effects of drop sets with resistance training on increases in muscle CSA, strength, and endurance: a pilot study. J. Sports Sci. 36, 691-696 (2018)

15. Poole DC, Jones AM: Measurement of the maximum oxygen uptake $\dot{V} \mathrm{O}_{2 \text { max }}: \dot{V} \mathrm{O}_{2 \text { peak }}$ is no longer acceptable. J. Appl. Physiol. (1985) 122, 997-1002 (2017)

16. Sanada K, Kearns CF, Kojima K, Abe T: Peak oxygen uptake during running and arm cranking normalized to total and regional skeletal muscle mass measured by magnetic resonance imaging. Eur. J. Appl. Physiol. 93, 687-693 (2005) 
17. Schoenfeld BJ, Peterson MD, Ogborn D, Contreras B, Sonmez GT: Effects of low- vs. high-load resistance training on muscle strength and hypertrophy in well-trained men. J. Strength Cond. Res. 29, 2954-2963 (2015)

18. Tabata I, Atomi Y, Kanehisa H, Miyashita M: Effect of high-intensity endurance training on isokinetic muscle power. Eur. J. Appl. Physiol. Occup. Physiol. 60, 254-258 (1990)

19. Tabata I, Irisawa K, Kouzaki M, Nishimura K, Ogita F, Miyachi M: Metabolic profile of high intensity intermittent exercises. Med. Sci. Sports Exerc. 29, 390-395 (1997)

20. Tabata I, Nishimura K, Kouzaki M, Hirai Y, Ogita F, Miyachi M, Yamamoto K: Effects of moderate-intensity endurance and high-intensity intermittent training on anaerobic capacity and $\dot{V} \mathrm{O}_{2 \max }$. Med. Sci. Sports Exerc. 28, 1327-1330 (1996)

21. Wenger HA, Bell GJ: The interactions of intensity, frequency and duration of exercise training in altering cardiorespiratory fitness. Sports Med. 3, 346-356 (1986) 